\title{
Prognostic impact of $\delta$-like ligand 4 and Notch1 in acute myeloid leukemia
}

\author{
JINGRU ZHANG $^{1 *}$, DAOXIN MA ${ }^{1 *}$, JINGJING YE $^{1 *}$, SHAOLEI ZANG ${ }^{1}$, FEI LU ${ }^{1}$, \\ MEIXIANG YANG ${ }^{2}, X \mathrm{XN}^{2}$, XIULIAN SUN ${ }^{3}$ and CHUNYAN JI ${ }^{1}$ \\ ${ }^{1}$ Department of Hematology, ${ }^{2}$ Institute of Basic Medical Sciences and ${ }^{3}$ National Key Laboratory \\ of Otolaryngology, Qilu Hospital, Shandong University, Jinan, P.R. China
}

Received May 16, 2012; Accepted July 3, 2012

DOI: $10.3892 /$ or.2012.1943

\begin{abstract}
Notch signaling plays a critical role in embryonic vascular development and tumor angiogenesis. The present study was conducted to investigate the prognostic role of the angiogenesis-related Notch ligand and the receptor in acute myeloid leukemia (AML) and assess whether their expression correlates with that of the vascular endothelial growth factor (VEGF) and angiopoietin (Ang)-2. Bone marrow mononuclear cells from 60 untreated AML patients and 40 healthy controls were obtained. Real-time RT-PCR was performed to evaluate the mRNA expression of $\delta$-like ligand 4 (Dll4), Notch1, VEGF, VEGF receptor (VEGFR)-1, VEGFR-2, Ang-1, Ang-2 and Tie2. Western blot analysis was used to determine the protein levels of D114 and Notch1. The results demonstrated that Dll4, Notch1, VEGF, VEGFR-2 and Ang-2 expression were significantly higher in untreated AML patients than in the controls. Univariate analysis of factors associated with the overall survival showed a significantly shorter survival in patients with the unfavorable karyotype, higher Dll4 expression, higher Notch1 expression, higher VEGF expression or higher Ang-2 expression. Furthermore, multivariate analysis revealed that the karyotype and expression levels of Notch1, Dl14, VEGF and Ang-2 were independent prognostic factors for overall survival. Additionally, the prognostic value of Dll4 expression (but not Notch1) was more significant in the subgroup consisting of patients with intermediate-risk cytogenetics. Subgroup analysis showed that Notch1 and Dll4 expression levels had a prognostic impact on patients with high VEGF or Ang-2 levels. Taken together, our data provide evidence that the activation of the Notch pathway may indicate an unfavorable prognosis in
\end{abstract}

Correspondence to: Dr Chunyan Ji, Department of Hematology, Qilu Hospital, Shandong University, 107 West Wenhua Road, Jinan 250012, P.R. China

E-mail: jichunyan@sdu.edu.cn

*Contributed equally

Key words: $\delta$-like ligand 4, Notch1, acute myeloid leukemia, angiogenesis, prognosis
AML. In particular, Dll4 may be a relevant prognostic marker in intermediate-risk AML.

\section{Introduction}

Angiogenesis, the process of new blood vessel formation from an existing network of vasculature, plays a key role in the growth and metastatic spread of solid tumors (1,2). Emerging evidence underscores the potential role of bone marrow (BM) vascularization, indicative of angiogenesis, in the progression and chemosensitivity of hematological malignancies, including acute myeloid leukemia (AML) $(3,4)$. Moreover, the BM microenvironment has been demonstrated to regulate the angiogenic switch and give rise to novel vascularization by controlling the balance between pro- and anti-angiogenic factors $(5,6)$. Among them, the vascular endothelial growth factor (VEGF) and angiopoietin (Ang) are 2 of the most important families involved in the regulation of angiogenesis (7).

The VEGF family is essential for the initiation of vascular development, while the Ang family is responsible for vascular maturation and stabilization (8). VEGF-A (from here on termed VEGF) is one of the most prominent angiogenic factors and it induces a pro-angiogenic and permeability-enhancing signal via the tyrosine kinase receptor, VEGFR-2 $(9,10)$. However, signaling through VEGFR-1 and the regulation of angiogenesis by this receptor is more complex $(9,10)$. Additionally, Ang-1 and -2 can bind to the Tie 2 receptor, although they have opposite effects on Tie2 activation $(11,12)$. Ang-1 functions as a stabilizing signal for mature vasculature, whereas Ang-2 may be regarded as a regulator of vessel plasticity (11). Dysregulation of the VEGF and Ang families is suggested to have a major impact on leukemic growth and has been established as a crucial step in the development of AML $(13,14)$. Indeed, elevated levels of VEGF and Ang-2 have been reported in patients with de novo AML and appear to be negative prognostic factors in AML (15-18).

The Notch pathway is an evolutionarily conserved intercellular pathway affecting a myriad of cellular activities, such as cell survival, proliferation, migration and invasion (19), and thereby may contribute to leukemogenesis $(20,21)$. Recently, the Notch pathway, particularly the vascular-specific $\delta$-like ligand 4 (Dll4), has been identified as another pivotal pathway in the regulation of vascular development (22). The activa- 
tion of the Notch pathway is initiated by ligand binding to Notch receptors mainly between adjacent cells, and there seems to be an important Notch cross-signaling between tumor and endothelial cells that promotes angiogenesis (23). Haploinsufficiency of Dll4 is essential for embryonic vascular development, and Dll4-mediated Notch signaling has a unique role in regulating endothelial cell proliferation and differentiation $(24,25)$. Studies have demonstrated that the Notch pathway displays either increased or decreased angiogenic processes, such as endothelial proliferation, migration and tube formation in vitro, depending on the cell type and context $(22,23)$. Furthermore, the prognostic impact of the Notch pathway in different malignancies has demonstrated a diverging pattern (26), and thus exploring the specific prognostic impact in AML angiogenesis is of substantial interest.

Previous studies have shown that VEGF and Ang act coordinately during vascular growth and remodeling (27). Moreover, the VEGF pathway acts as a potent upstream activating stimulus for angiogenesis, while the Notch/Dll4 pathway contributes to guide cell fate decisions that shape the activation appropriately $(28,29)$. In addition, Notch1 signaling activates the Ang pathway, presenting that angiogenesis of endothelial cells is mediated in part through a Notch-dependent Ang-1/Tie2 pathway (30). In turn, Ang-1/Tie2 signaling potentiates basal Notch1 signaling controlling vascular quiescence by upregulating Dll4 (31). Since BM neovascularization depends on the mutual and coordinated interaction of various angiogenic factors from endothelial cells and leukemia cells $(4,6,32)$, and many of these factors that are present in significant amounts in the BM microenvironment may function in a synchronized fashion, it will be of interest to comprehensively analyze the correlation among these factors and further assess the clinical implications.

In this study, we investigated the expression of 8 angiogenesis-related factors (D114, Notch1, VEGF, VEGFR-1, VEGFR-2, Ang-1, Ang-2 and Tie2) in BM mononuclear cells of adult patients with untreated AML. Furthermore, we assessed the prognostic impact of Dll4 and Notch1 expression levels and their association with the VEGF and Ang families.

\section{Materials and methods}

Patient samples. Sixty adult patients with untreated AML (31 males and 29 females; median age, 45 years; range, 14-73 years) were enrolled in this study. Diagnoses were established according to the French-American-British (FAB) criteria (33). All the patients with non-M3 AML subtypes underwent standard induction chemotherapy with one of the anthracyclines (doxorubicin or idarubicin) for 3 days and cytarabine for 7 days. The patients with acute promyelocytic leukemia (subtype M3) received all-trans retinoic acid with or without concurrent induction chemotherapy. After the patients achieved complete remission (CR), they underwent consolidation chemotherapy with a conventional dose of cytarabine and one anthracycline or with a high dose of cytarabine. The control group consisted of 40 healthy adult donors (22 males and 18 females; median age, 44 years; range, 24-68 years), with normal BM morphology as demonstrated by cytological and histological analyses. Chromosomal analyses for newly diagnosed patients were conducted on BM cells after 1-3 days of unstimulated culture and karyotyped according to the International System for
Table I. Clinical features for AML patients

\begin{tabular}{|c|c|}
\hline Features & Patients \\
\hline No. & 60 \\
\hline Median age (range, years) & $45(14-73)$ \\
\hline \multicolumn{2}{|l|}{ Gender } \\
\hline Male & 31 \\
\hline Female & 29 \\
\hline \multicolumn{2}{|l|}{ FAB classification } \\
\hline M0 & 2 \\
\hline M1 & 3 \\
\hline M2 & 12 \\
\hline M3 & 12 \\
\hline M4 & 10 \\
\hline M5 & 16 \\
\hline M6 & 5 \\
\hline Median hemoglobin (range, g/l) & $75.5(31.0-122.0)$ \\
\hline Median WBC (range, $\left.10^{9} / 1\right)$ & $16.0(0.3-110.3)$ \\
\hline Median PLT (range, $10^{9} / 1$ ) & $43.5(3.0-836.0)$ \\
\hline Median BM blasts (range, \%) & $70(40-98)$ \\
\hline \multicolumn{2}{|l|}{ Karyotype } \\
\hline Favorable: $\mathrm{t}(8 ; 21), \mathrm{t}(15 ; 17), \operatorname{inv}(16)$ & 16 \\
\hline Intermediate: normal, $+8,+22$, other & 39 \\
\hline Unfavorable: $-7,-5$, complex & 5 \\
\hline Median follow-up (range, months) & $25.0(7.0-45.9)$ \\
\hline
\end{tabular}

AML, acute myeloid leukemia; FAB, French-American-British classification; WBC, white blood cells; PLT, platelet; BM, bone marrow.

Human Cytogenetic Nomenclature (ISCN). The median followup duration was 25 months. This study protocol was approved by the Medical Ethics Committee of the Affiliated Qilu Hospital of Shandong University, Jinan, China, and written informed consent was obtained from all patients. The clinical information for the 60 AML patients is presented in Table I.

Real-time reverse transcription-PCR (RT-PCR) analysis. Total RNA was isolated using TRIzol (Invitrogen, Carlsbad, CA, USA) and cDNA was prepared using M-MLV Reverse Transcriptase (Promega, Madison, WI, USA) according to the manufacturer's instructions. Real-time RT-PCR was carried out using an ABI Prism 7500 sequence detection system (Applied Biosystems, Foster City, CA, USA) and performed with SYBRGreen PCR Master Mix (Toyobo, Osaka, Japan) in a $20 \mu \mathrm{l}$ reaction volume as described previously (34). The specificity of the desired products was documented with melting curves analysis and examined using electrophoresis on a $2 \%$ agarose gel. Each sample was tested twice, while PCR-grade water instead of template cDNA was used as the negative controls. A comparative CT method $\left(2^{-\Delta \Delta C T}\right)$ was used to analyze the relative changes in gene expression. The results were expressed relative to the number of GAPDH transcripts used as the internal control. The sequences of primers of different angiogenic factors are listed in Table II. 
Table II. Primer sequences used for real-time RT-PCR.

\begin{tabular}{|c|c|c|c|}
\hline ID & Genes & Primers $\left(5^{\prime}-3^{\prime}\right)$ & Size \\
\hline NM_017617.3 & Notch1 & $\begin{array}{l}\text { F: TCAGCGGGATCCACTGTGAG } \\
\text { R: ACACAGGCAGGTGAACGAGTTG }\end{array}$ & 104 \\
\hline NM_019074.3 & Dl14 & $\begin{array}{l}\text { F: GCCAACTATGCTTGTGAATGTCC } \\
\text { R: CAGTAGGTGCCCGTGAATCC }\end{array}$ & 161 \\
\hline NM_001025366.2 & VEGF & $\begin{array}{l}\text { F: GAGCCTTGCCTTGCTGCTCTAC } \\
\text { R: CACCAGGGTCTCGATTGGATG }\end{array}$ & 148 \\
\hline NM_002019.4 & VEGFR-1 & $\begin{array}{l}\text { F: CTGGACTGACAGCAAACCCAAG } \\
\text { R: CCACAGCTGGAATGGCAGAA }\end{array}$ & 117 \\
\hline NM_002253.2 & VEGFR-2 & $\begin{array}{l}\text { F: AGCCAGCTCTGGATTTGTGGA } \\
\text { R: CATGCCCTTAGCCACTTGGAA }\end{array}$ & 133 \\
\hline NM_001146.3 & Ang-1 & $\begin{array}{l}\text { F: CCTGATCTTACACGGTGCTGATT } \\
\text { R: GTCCCGCAGTATAGAACATTCCA }\end{array}$ & 146 \\
\hline NM_001147.2 & Ang-2 & $\begin{array}{l}\text { F: AAGAGATCAAGGCCTACTGTGACA } \\
\text { R: TCCTCACGTCGCTGAATAATTG }\end{array}$ & 70 \\
\hline NM_000459.3 & Tie2 & $\begin{array}{l}\text { F: CTGTGAAGGGCGAGTTCGA } \\
\text { R: TGGTAGGAAGGAAGCTTGTTGAC }\end{array}$ & 76 \\
\hline NM_002046.3 & GAPDH & $\begin{array}{l}\text { F: GCACCGTCAAGGCTGAGAAC } \\
\text { R: TGGTGAAGACGCCAGTGGA }\end{array}$ & 138 \\
\hline
\end{tabular}

F, forward; R, reverse; Dll4, $\delta$-like ligand 4; VEGF, vascular endothelial growth factor; VEGFR, vascular endothelial growth factor receptor; Ang, angiopoietin; GAPDH, glyceraldehyde-3-phosphate dehydrogenase.

Western blot analysis for Dll4 and Notch1 protein. The cells were lysed in lysis buffer [ $50 \mathrm{mmol} / \mathrm{l}$ Tris (pH 7.5), $100 \mathrm{mmol} / 1$ $\mathrm{NaCl}, 1 \mathrm{mmol} / \mathrm{l}$ EDTA, 0.5\% NP40, 0.5\% Triton X-100, $2.5 \mathrm{mmol} / \mathrm{l}$ sodium orthovanadate, $10 \mu \mathrm{l} / \mathrm{ml}$ protease inhibitor cocktail, $1 \mathrm{mmol} / 1$ phenylmethylsulfonyl fluoride] for $30 \mathrm{~min}$ at $4^{\circ} \mathrm{C}$. Total proteins were fractionated using SDS-PAGE and were then transferred onto nitrocellulose membranes. The membranes were then incubated with the appropriate primary antibodies (Dl14 and Notch1), followed by incubation with a secondary HRP-conjugated antibody (Jingmei Co., Ltd., Beijing, China). The probed proteins were detected using enhanced chemiluminescent reagents (Amersham Pharmacia Biotech, Piscataway, NJ, USA). The two-dimensional optical densities of proteins on the film were quantified and analyzed using the Quantity One software (Bio-Rad, Hercules, CA, USA).

Statistical analysis. The Mann-Whitney U test, analysis of variance (ANOVA) and the Kruskal-Wallis test were used to analyze the differences in the expression of angiogenic factors between the AML and control groups, as well as differences in age, gender, FAB subtype and karyotype subgroups of the AML patients. Spearman's test was used to evaluate the correlation between the individual expression of the genes studied, and the association of gene expression with clinical features. Furthermore, overall survival (OS) was defined as the number of days from the date of the first diagnosis to death from any cause. Patients were categorized into high and low angiogenic factors expressing subgroups using the median value as the cut-off. Kaplan-Meier estimation was applied to plot survival curves, and a log-rank test was used to compare survival between groups. Univariate Cox proportional hazards regression models were used to evaluate the predictive effect of each factor alone on survival. Factors statistically significant in the univariate models at a 5\% level were included in the multivariate models. Subsequently, multivariate models were reduced one factor at a time such that all factors remaining in the model were statistically significant at a $5 \%$ significance level. Multivariate Cox models estimated the hazard ratio in terms of relative risk (RR) for death, and $95 \%$ confidence interval (CI) to determine independent risk factors associated with survival. Two-sided $\mathrm{P}<0.05$ were considered statistically significant. All statistical analyses were performed with the SPSS 13 software (SPSS Inc., Chicago, IL, USA).

\section{Results}

Aberrant expression profile of angiogenic factors in $A M L$ patients. As a ratio with the expression of the housekeeping gene GAPDH, BM levels of the 8 angiogenesis-related factors were compared between the untreated AML patients and the healthy controls. In spite of the wide range of individual values, median levels of Notch1 $(\mathrm{P}=0.012)$, Dl14 $(\mathrm{P}=0.001)$, VEGF ( $\mathrm{P}=0.005)$, VEGFR-2 ( $\mathrm{P}=0.009)$ and Ang-2 ( $\mathrm{P}=0.006)$ were significantly elevated in the AML patients compared with the controls, while the median levels of VEGFR-1 ( $\mathrm{P}=0.439)$, Ang-1 $(\mathrm{P}=0.123)$ and Tie2 $(\mathrm{P}=0.733)$ were similar in $\mathrm{AML}$ and control patients (Fig. 1A). 

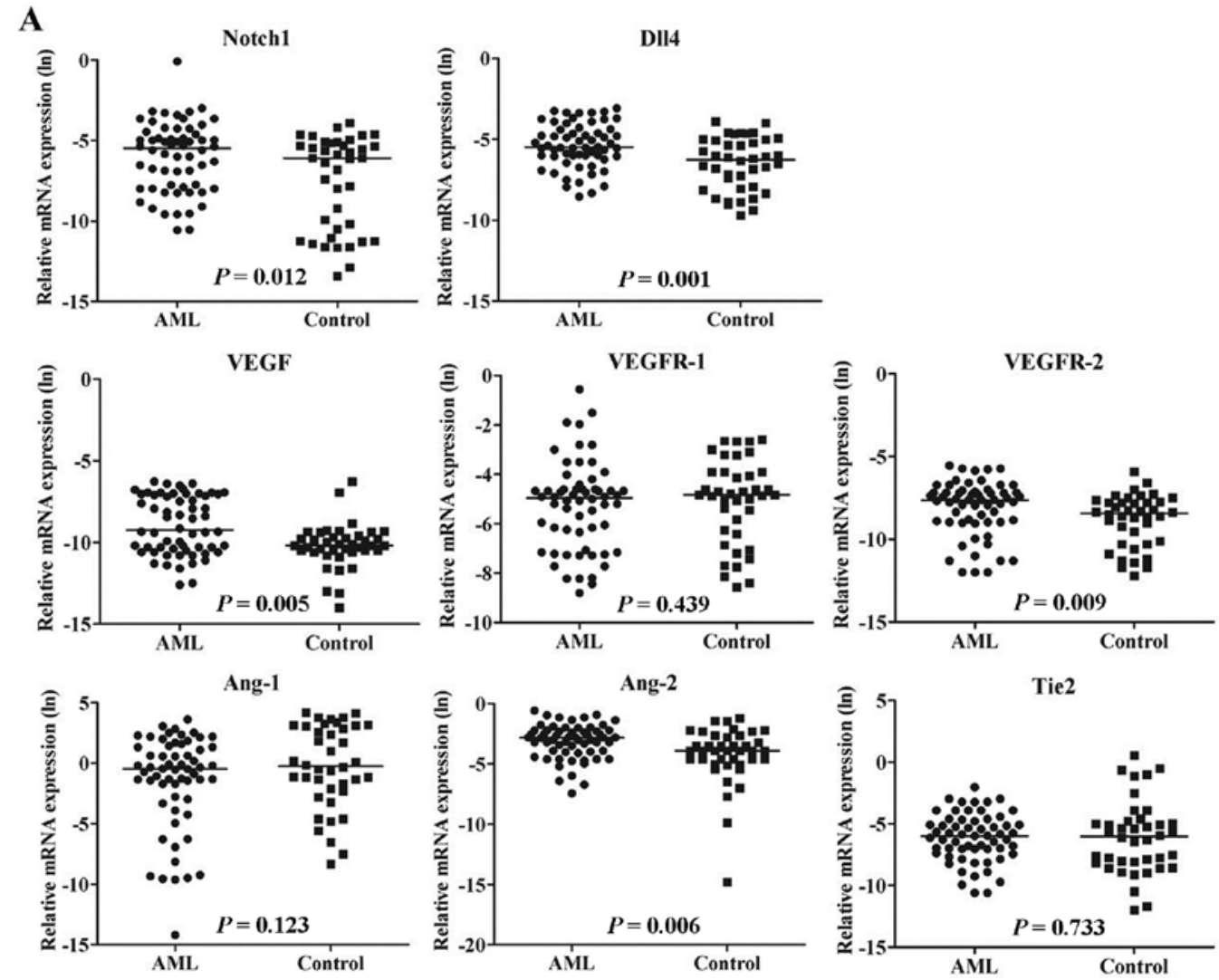

B
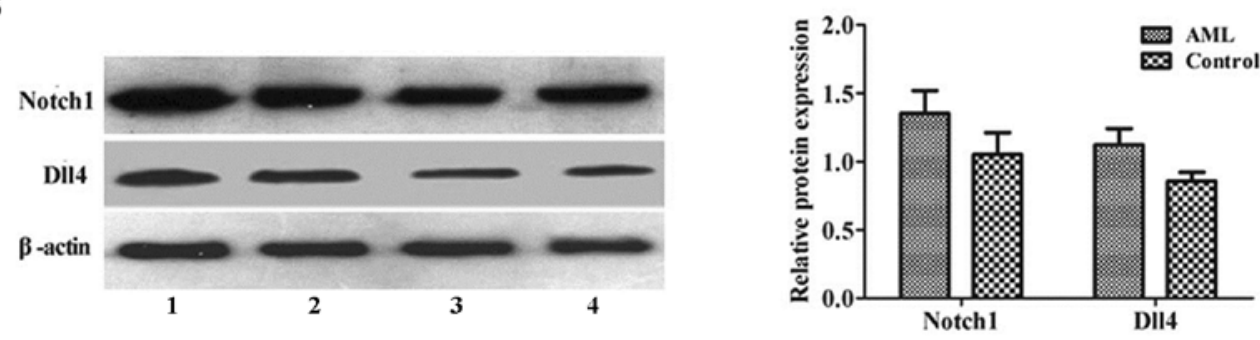

Figure 1. Aberrant expression profile of angiogenic factors in AML patients. (A) Real-time RT-PCR analysis of Notch1, Dl14, VEGF, VEGFR-1, VEGFR-2, Ang-1, Ang-2 and Tie2 expression in 60 untreated AML patients and 40 healthy controls. The mRNA expressions of Notch1, Dll4, VEGF, VEGFR-2 and Ang-2 were significantly upregulated in BM leukemia cells from AML patients, compared to the controls. GAPDH was used to normalize the mRNA level. Solid points indicate individual values and horizontal lines represent the group median. Data were analyzed using the Mann-Whitney U test. (B) Western blot analysis of Notch1 and Dl14 expression in 24 untreated AML patients and 8 healthy controls. Left panel, representative photomicrographs of western blot analysis of Notch1 and D114. Lane 1, AML patient no. 1; lane 2, AML patient no. 2; lane 3, control no. 7; and lane 4, control no. 8 . $\beta$-actin was used as the sample loading control. Right panel, the protein expressions of Notch1 and Dll4 were significantly higher in BM leukemia cells from AML patients compared to the controls $(\mathrm{P}<0.001)$. Columns indicate the mean from 2 independent experiments and bars represent SD. Data were analyzed using the Student's t-test.

It is important to note that the active functional form of Notch1 is the Notch1 intracellular domain. Therefore, we focused our study on the Notch1 intracellular domain at the protein level and Notch1 in the figure legends indicates the active functional form of Notch1. In concordance with the real-time RT-PCR data, western blot analysis showed that the protein expression levels of Notch1 and Dll4 were upregulated in the untreated AML patients compared to the controls $(\mathrm{P}<0.001$, Fig. 1B). Taken together, these data indicate that the Notch/Dll4 pathway is aberrantly activated in AML.

It has been reported that BM vascularization is regulated by the relative balance between angiogenic activators and inhibitors $(4,35)$. Therefore, we investigated the correlation between the angiogenic factors studied in AML patients. Significantly positive correlations were observed between Notch1 and Dll4 $(\mathrm{r}=0.326 ; \mathrm{P}=0.013), \mathrm{VEGF}$ and VEGFR-2 ( $\mathrm{r}=0.354 ; \mathrm{P}=0.007)$ and Ang-1 and Tie2 ( $\mathrm{r}=0.463 ; \mathrm{P}=0.001)$. Furthermore, Dll4 was closely associated with VEGF $(\mathrm{r}=0.663 ; \mathrm{P}=0.001)$ and Notch1 correlated well with Ang-2 ( $\mathrm{r}=0.324 ; \mathrm{P}=0.012)$.

VEGF receptors expressed in leukemic cells are functional and convey distinct signals, such as increasing proliferation, metalloproteinase activation and trans-membrane migration (9). The degree of Tie 2 receptor activation depends on the balance between agonistic and antagonistic ligands (11). Thus, we further calculated the VEGFR-2:VEGFR-1 and Ang-2:Ang-1 expression ratio in normal and leukemic cells. The median VEGFR-2:VEGFR-1 expression ratio was 3.61-fold higher in the AML patients than in the controls $(\mathrm{P}=0.04)$, suggesting that the pathway enhanced by the VEGF/ VEGFR-2 interaction may be essential for the pathogenesis of 
Table III. Expression of angiogenic factors in the different subgroups.

\begin{tabular}{|c|c|c|c|c|c|c|c|c|c|}
\hline Features & No. & Notch1 & Dll4 & VEGF & VEGFR-1 & VEGFR-2 & Ang-1 & Ang-2 & Tie2 \\
\hline \multicolumn{10}{|l|}{ Age (years) } \\
\hline$<45$ & 30 & $0.62 \pm 1.36$ & $0.39 \pm 1.26$ & $0.85 \pm 4.94$ & $0.66 \pm 5.22$ & $0.45 \pm 1.08$ & $0.87 \pm 7.82$ & $0.55 \pm 0.78$ & $0.50 \pm 2.60$ \\
\hline$\geq 45$ & 30 & $0.16 \pm 16.53$ & $0.48 \pm 1.06$ & $1.51 \pm 5.17$ & $0.79 \pm 10.3$ & $0.50 \pm 0.64$ & $0.31 \pm 4.34$ & $0.60 \pm 1.35$ & $0.13 \pm 1.01$ \\
\hline $\mathrm{P}$-value & & 0.109 & 0.631 & 0.584 & 0.971 & 0.953 & 0.191 & 0.652 & $0.030^{\mathrm{a}}$ \\
\hline \multicolumn{10}{|l|}{ Gender } \\
\hline Male & 31 & $0.47 \pm 16.22$ & $0.52 \pm 1.20$ & $0.86 \pm 5.33$ & $0.74 \pm 10.7$ & $0.43 \pm 0.88$ & $0.91 \pm 7.82$ & $0.63 \pm 1.19$ & $0.26 \pm 1.18$ \\
\hline Female & 29 & $0.37 \pm 0.95$ & $0.40 \pm 1.11$ & $1.96 \pm 4.74$ & $0.54 \pm 3.64$ & $0.49 \pm 0.91$ & $0.26 \pm 3.91$ & $0.57 \pm 1.04$ & $0.19 \pm 2.64$ \\
\hline P-value & & 0.383 & 0.717 & 0.912 & 0.549 & 0.510 & 0.061 & 0.935 & 0.745 \\
\hline \multicolumn{10}{|l|}{ FAB subtypes } \\
\hline M0 & 2 & $4.35 \pm 0.91$ & $0.26 \pm 0.19$ & $0.54 \pm 0.39$ & $1.10 \pm 3.68$ & $1.16 \pm 0.61$ & $0.46 \pm 0.63$ & $0.87 \pm 0.33$ & $0.19 \pm 0.04$ \\
\hline M1 & 3 & $2.60 \pm 1.90$ & $1.25 \pm 1.10$ & $1.06 \pm 8.11$ & $0.08 \pm 0.42$ & $0.12 \pm 0.32$ & $0.02 \pm 0.51$ & $2.48 \pm 1.40$ & $0.12 \pm 0.31$ \\
\hline M2 & 12 & $0.76 \pm 26.03$ & $0.70 \pm 1.28$ & $3.25 \pm 4.19$ & $0.63 \pm 6.18$ & $0.48 \pm 1.04$ & $2.47 \pm 11.3$ & $0.51 \pm 0.53$ & $0.39 \pm 1.14$ \\
\hline M3 & 12 & $0.20 \pm 0.51$ & $0.26 \pm 0.53$ & $0.25 \pm 2.47$ & $0.36 \pm 5.48$ & $0.30 \pm 0.43$ & $2.20 \pm 4.69$ & $0.45 \pm 1.26$ & $0.36 \pm 3.75$ \\
\hline M4 & 10 & $0.10 \pm 0.48$ & $0.31 \pm 1.04$ & $2.78 \pm 5.27$ & $0.85 \pm 1.76$ & $0.49 \pm 1.16$ & $0.01 \pm 0.25$ & $0.66 \pm 1.17$ & $0.09 \pm 1.62$ \\
\hline M5 & 16 & $0.47 \pm 1.19$ & $0.48 \pm 1.52$ & $5.25 \pm 6.22$ & $0.73 \pm 0.53$ & $0.58 \pm 0.82$ & $1.75 \pm 4.53$ & $0.45 \pm 1.34$ & $0.25 \pm 1.32$ \\
\hline M6 & 5 & $0.70 \pm 1.06$ & $0.98 \pm 0.81$ & $0.36 \pm 4.09$ & $0.34 \pm 2.56$ & $0.61 \pm 1.29$ & $0.34 \pm 3.14$ & $0.62 \pm 0.99$ & $0.32 \pm 0.38$ \\
\hline P-value & & 0.661 & 0.474 & 0.258 & 0.53 & 0.725 & 0.140 & 0.676 & 0.595 \\
\hline \multicolumn{10}{|l|}{ Karyotype } \\
\hline Favorable & 16 & $0.11 \pm 0.25$ & $0.25 \pm 0.29$ & $0.29 \pm 0.73$ & $0.50 \pm 6.32$ & $0.36 \pm 0.73$ & $2.79 \pm 4.27$ & $0.35 \pm 0.76$ & $0.22 \pm 3.31$ \\
\hline Intermediate & 39 & $0.52 \pm 14.47$ & $0.45 \pm 1.15$ & $3.60 \pm 4.98$ & $0.74 \pm 9.24$ & $0.61 \pm 0.97$ & $0.49 \pm 7.23$ & $0.63 \pm 1.01$ & $0.26 \pm 1.29$ \\
\hline Unfavorable & 5 & $2.20 \pm 1.37$ & $2.00 \pm 1.34$ & $11.36 \pm 6.0$ & $0.63 \pm 0.34$ & $0.52 \pm 0.55$ & $0.62 \pm 5.23$ & $0.92 \pm 2.21$ & $0.29 \pm 0.81$ \\
\hline P-value & & 0.676 & $0.001^{\mathrm{a}}$ & $0.001^{\mathrm{a}}$ & 0.835 & 0.510 & 0.917 & 0.092 & 0.319 \\
\hline
\end{tabular}

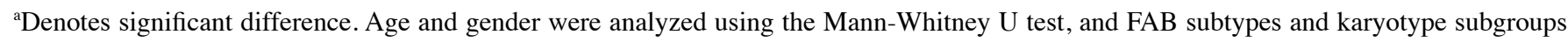
were analyzed using ANOVA and the Kruskall-Wallis test. FAB, French-American-British classification; ANOVA, analysis of variance.

AML. The median Ang-2:Ang-1 expression ratio was 3.18-fold higher in the AML patients than in the controls $(\mathrm{P}=0.015)$, indicating a dominant influence of Ang-2 in the neoplastic BM and Ang-1 in the normal BM microenvironment.

Correlation of angiogenic factor expression with clinical features. The association between BM levels of these 8 angiogenic factors in the $60 \mathrm{AML}$ patients and clinical features were investigated, and significant differences were not observed in the gender and FAB subtypes (Table III). However, the Tie-2 level was higher in AML patients $<45$ years old than those $>45$ years old $(\mathrm{P}=0.03)$, and the levels of Dll4 and VEGF were significantly different among the karyotype subgroups $(\mathrm{P}=0.001)$. The $\mathrm{BM}$ of the AML patients studied was highly infiltrated by leukemic blasts. The median (range) percentage of blasts was $70 \%$ (range 40-98\%). Significantly positive associations of the percentage of leukemic blast infiltration were observed with the individual expression of Notch1 $(\mathrm{r}=0.428$; $\mathrm{P}=0.001)$, Dll4 ( $\mathrm{r}=0.288 ; \mathrm{P}=0.026)$, VEGF $(\mathrm{r}=0.428 ; \mathrm{P}=0.001)$ and Ang-2 ( $\mathrm{r}=0.474 ; \mathrm{P}<0.001)$.

Association between angiogenic factor expression and clinical outcome. We subsequently investigated the correlation between pre-therapeutic angiogenic factor expression and prognosis in AML. The median OS was 25 months (range, 7.0-45.9 months).
Univariate analysis of the factors associated with OS revealed a significantly shorter survival in the patients with the unfavorable karyotype, higher Notch1 expression, higher D114 expression, higher VEGF expression or higher Ang-2 expression (Table IV). Variables, such as age, gender, hemoglobin levels, white blood cell (WBC) counts, platelet counts, VEGFR-1, VEGFR-2, Ang-1 and Tie2 expression, as well as VEGFR-2:VEGFR-1 and Ang-2:Ang-1 expression ratio had no impact. Cox proportional hazards multivariate analysis of the univariate predictors identified karyotype (RR, 3.19; 95\% CI, 1.18-8.63; $\mathrm{P}=0.22)$ and gene expression of Notch1 (RR, 5.04; 95\% CI, 1.62-15.69; P=0.005), Dll4 (RR, 8.09; 95\% CI, 1.72-37.95; $\mathrm{P}=0.008)$, VEGF (RR, 5.25; 95\% CI, 1.17-23.48; $\mathrm{P}=0.03)$ and Ang-2 (RR, 11.25; 95\% CI, 3.09-41.01; $\mathrm{P}=0.001)$ as prognostic factors for OS. The Kaplan-Meier curves for OS stratified according to Notch1, Dll4, VEGF and Ang-2 expression in BM leukemia cells are shown in Fig. 2A-D.

In order to further illustrate the impact of Notch1 and Dll4 expression on OS, the Kaplan-Meier survival curves of 3 cytogenetic-risk groups were analyzed. Dl14 expression had a similar impact on OS in all the patients and those with the intermediate-risk karyotype, whereas Notch1 expression lost its predictive value for survival in patients with the intermediate-risk karyotype (Fig. 2E and F). However, there was no survival difference between patients with a low and 
Table IV. Univariate analysis of the impact of variables on overall survival.

\begin{tabular}{|c|c|c|c|}
\hline Variables $^{\mathrm{a}}$ & $\begin{array}{l}\text { No. of } \\
\text { patients }\end{array}$ & $\begin{array}{c}\text { Median } \\
(\text { months } \pm \mathrm{SD})\end{array}$ & P-value \\
\hline Age (years) & & & 0.837 \\
\hline$<45$ & 30 & $25.5 \pm 10.2$ & \\
\hline$\geq 45$ & 30 & $24.6 \pm 9.8$ & \\
\hline Gender & & & 0.532 \\
\hline Female & 31 & $26.8 \pm 11.0$ & \\
\hline Male & 29 & $24.8 \pm 8.7$ & \\
\hline Karyotype & & & 0.001 \\
\hline Favorable & 16 & $37.9 \pm 7.1$ & \\
\hline Intermediate & 39 & $22.7 \pm 8.0$ & \\
\hline Unfavorable & 5 & $13.7 \pm 3.9$ & \\
\hline Notch1 & & & 0.004 \\
\hline Low & 30 & $29.5 \pm 9.1$ & \\
\hline High & 30 & $19.5 \pm 8.5$ & \\
\hline Dl14 & & & 0.001 \\
\hline Low & 30 & $34.5 \pm 8.0$ & \\
\hline High & 30 & $17.4 \pm 5.8$ & \\
\hline VEGF & & & 0.001 \\
\hline Low & 30 & $34.5 \pm 9.0$ & \\
\hline High & 30 & $19.4 \pm 5.9$ & \\
\hline VEGFR-1 & & & 0.090 \\
\hline Low & 30 & $26.5 \pm 10.2$ & \\
\hline High & 30 & $24.6 \pm 9.8$ & \\
\hline VEGFR-2 & & & 0.149 \\
\hline Low & 30 & $28.7 \pm 9.4$ & \\
\hline High & 30 & $22.0 \pm 10.2$ & \\
\hline Ang-1 & & & 0.865 \\
\hline Low & 30 & $23.6 \pm 9.7$ & \\
\hline High & 30 & $25.9 \pm 10.3$ & \\
\hline Ang-2 & & & 0.005 \\
\hline Low & 30 & $29.5 \pm 9.3$ & \\
\hline High & 30 & $19.5 \pm 8.7$ & \\
\hline Tie2 & & & 0.955 \\
\hline Low & 30 & $26.1 \pm 9.8$ & \\
\hline High & 30 & $24.8 \pm 10.2$ & \\
\hline
\end{tabular}

${ }^{a}$ Median values of each variable were used as the cut-off levels to define low and high expression subgroups.

high expression of Notch1 or D114 in the favorable-risk group and unfavorable-risk group.

Finally, we performed subgroup analyses to investigate potential interactions between the Notch/D1l4 pathway and other angiogenic factors on OS. Patients were divided into 2 groups with a low or high expression (respectively, below or above the median level) of VEGF and Ang-2. Survival estimates were calculated for each group according to the Notch1 and Dll4 expression levels. The survival difference between patients with a low and high Notch1 expression was pronounced in the subgroups with high VEGF levels $(\mathrm{P}=0.001)$ and high Ang-2 levels $(\mathrm{P}=0.001)$, respectively, but not evident in the patients with low values (Fig. 3A and B). However, the outcome was insignificant in patients with a high compared to a low Dll4 expression in the presence of high VEGF expression $(\mathrm{P}=0.001$, Fig. 3C). The impact of the Dll4 expression on OS was equally prominent in the subgroups of AML patients expressing low and high levels of Ang-2 ( $\mathrm{P}=0.001$, Fig. 3D).

\section{Discussion}

During the past few years, it has been proposed that AML may be an angiogenesis-dependent disease with progressive recruitment of blood vessels in the BM microenvironment (35). Previous studies have demonstrated a close correlation between leukemogenesis and dysregulation of angiogenesis, including increased BM vascularity (3) and elevated levels of numerous angiogenic factors $(7,13)$. Our present study clearly demonstrates an elevated expression of Notch1, Dll4, VEGF, VEGFR-2 and Ang-2 in the BM leukemia cells of patients with untreated AML, compared with the controls. Cox regression analyses revealed that the expression levels of Notch1, Dll4, VEGF and Ang-2 were predictors of poor OS, independent of cytogenetics. Furthermore, the prognostic relevance of Dll4 expression was more evident in patients with the intermediate-risk karyotype (62.5\% of patients with low Dll4 expression vs. $33.3 \%$ with high level in this subgroup survived, $\mathrm{P}=0.001$ ). Therefore, the Dll4 expression may serve as a potential biomarker for prognosis prediction, particularly in the intermediate-risk cytogenetic group.

Aberrant Notch signaling contributes to the genesis of diverse cancers (26). The fact that constitutively active Notch1 transforms cells in vitro and causes mice to develop leukemia indicates the Notch pathway involvement in the development of leukemia (21). Conversely, Notch is a tumor suppressor in certain epithelial cancers where its normal function is to promote terminal differentiation (36). Our present study shows that patients with untreated AML express higher levels of Notch1 and Dll4 compared to healthy controls, as assessed by real-time RT-PCR and western blot analysis. Moreover, the high expression levels of Notch1 and Dll4 represent a significant feature of AML patients. Patients with high Notch1 and Dll4 expression levels show several adverse prognostic indicators, such as high BM blast counts and unfavorable karotype. Recent studies have shown that reciprocal Notch signaling may be necessary for the proliferation and survival of AML cells, possibly through the maintenance of $\mathrm{c}-\mathrm{Myc}$ and $\mathrm{Bcl} 2$, as well as the phosphorylation of $\mathrm{Rb}$ protein $(37,38)$. Furthermore, the activation of the Notch pathway has been reported to be associated with an adverse outcome in various solid cancers, such as lung (39), breast (40) and bladder cancer (41). Consistent with these findings, our study also demonstrates that there is an inverse correlation between Notch1/Dll4 expression and OS in AML. Collectively, our data provide evidence for the critical role of the Notch/Dll4 pathway activation in the pathogenesis and prognosis of AML.

Elegant genetic and molecular studies have suggested that the Notch/D114 pathway interacts extensively with the VEGF pathway in endothelial cells $(42,43)$. Indeed, Dll4 expression 
A

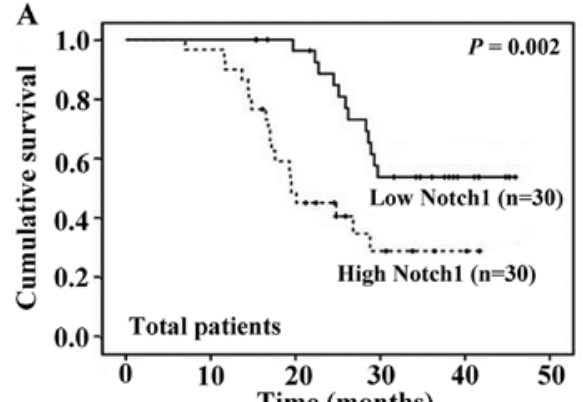

C

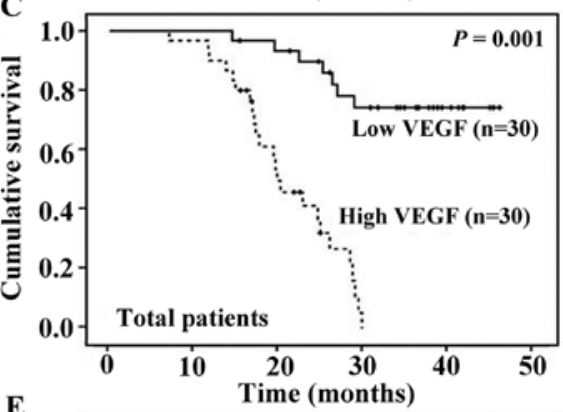

E

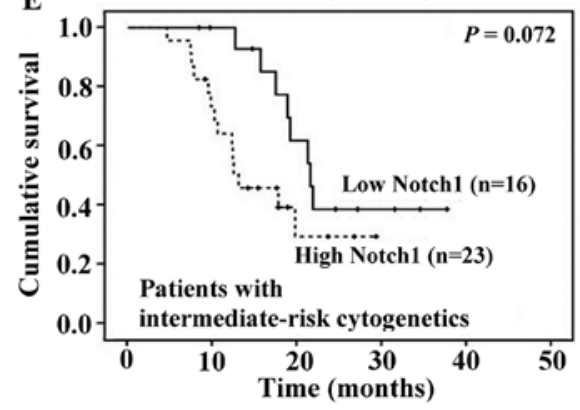

B

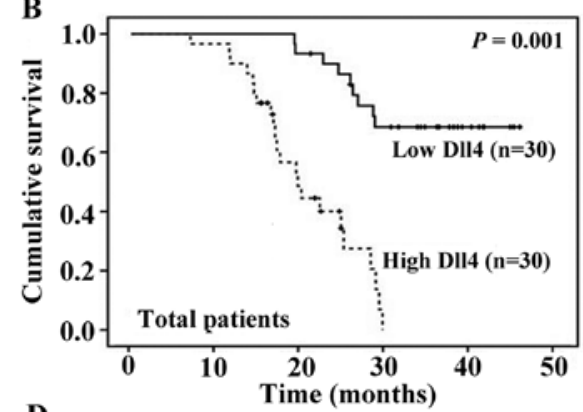

D
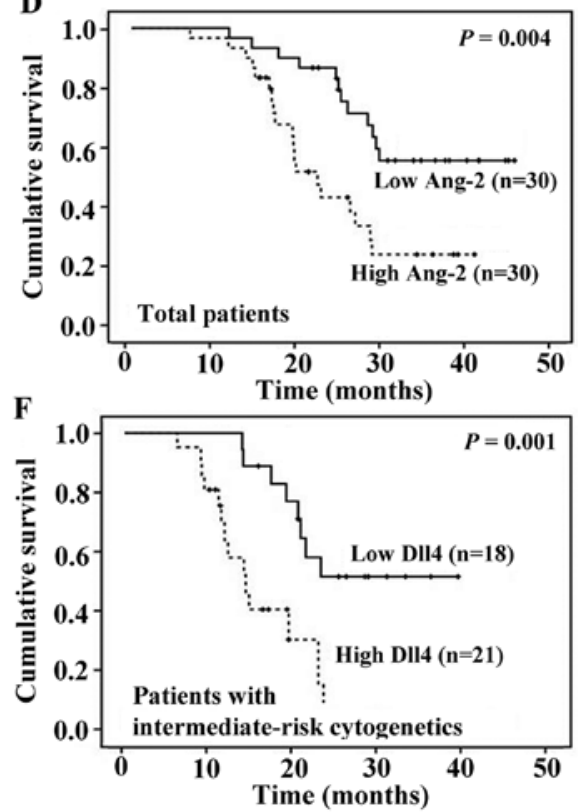

Figure 2. Kaplan-Meier survival plots for overall survival of all patients with untreated AML stratified by the expression of (A) Notch1, (B) D114, (C) VEGF and (D) Ang-2. Kaplan-Meier survival plots for overall survival of patients with intermediate-risk cytogenetics stratified by the expression of (E) Notch1 and (F) D114. Data were analyzed using the log-rank test.

may be induced by VEGF and hypoxia (42). Conversely, Dll4 is considered as a selective inhibitor of VEGF biological activity by downregulating VEGFR-2 (43). Recently, emphasis has been placed on the interaction of the Notch/Dll4 and VEGF pathways in tumor angiogenesis $(28,29)$. Our study shows that Dll4 positively correlates with VEGF in AML patients. Moreover, the poor prognosis in patients with high Notch1 and D114 expression levels was only evident in the presence of higher (not lower) levels of VEGF, suggesting that the angiogenic effect of Notch/Dl14 pathway may be dependent on VEGF. Donnem et al also reported that Notch1 and Dll4 were independent prognostic factors for non-small cell lung cancer, and the influence of Notch1 was enhanced in the presence of high VEGF expression (39). Thus, our results and published reports confirm the hypothesis of a cross-talk between Notch/ DIl4 and VEGF pathways in tumor angiogenesis.

Notch1 signaling has been shown to activate the Ang pathway, and Ang-1/Tie2 signaling augments basal Notch signaling by inducing Dll4 expression through the Akt-mediated activation of $\beta$-catenin $(30,31)$. The fact that Notch1 lost its impact on survival in the patients with low Ang- 2 expression, as revealed in our study, suggests these 2 factors may function in a synergetic fashion; however, the mechanism involved remains unclear. The grade of Tie 2 phosphorylation is determined by the balance between Ang-1 and its naturally occurring antagonist Ang-2 (11). Owing to low Ang-2 expression, Ang-1-mediated Tie2 activation was relatively enhanced, resulting in the activation of the Akt pathway, which is associated with increased survival and the anti-apoptotic effect $(44,45)$. Therefore, the prognostic impact of Notch1 expression may be lessened in the presence of low Ang-2 expression.

Previous studies have led to the proposal that VEGFR-2 is the critical receptor for transmitting cellular signals for the proliferation and differentiation of endothelial cells, whereas VEGFR-1 may be more important for vascular remodeling and monocyte migration (9). Additionally, several studies have shown that Ang-1 mediates vascular stability, while Ang-2 induces vascular instability by overriding Ang-1-mediated Tie 2 activation (11). Therefore, it is important to elucidate the balance between VEGFR-1 and VEGFR-2, Ang-1 and Ang-2 in the BM microenvironment. In this study, we observed a difference in the VEGFR-2:VEGFR-1 and Ang-2:Ang-1 expression ratio between $A M L$ patients and healthy controls, indicating that the angiogenic balance is altered in favor of VEGFR-2 and Ang-2 in AML. Indeed, a high ratio of Ang-2:Ang-1 expression in association with high levels of VEGF, bFGF and other mitogenic angiogenic factors stimulates angiogenesis in a wide range of solid tumors (11). Thus, our data provide evidence 

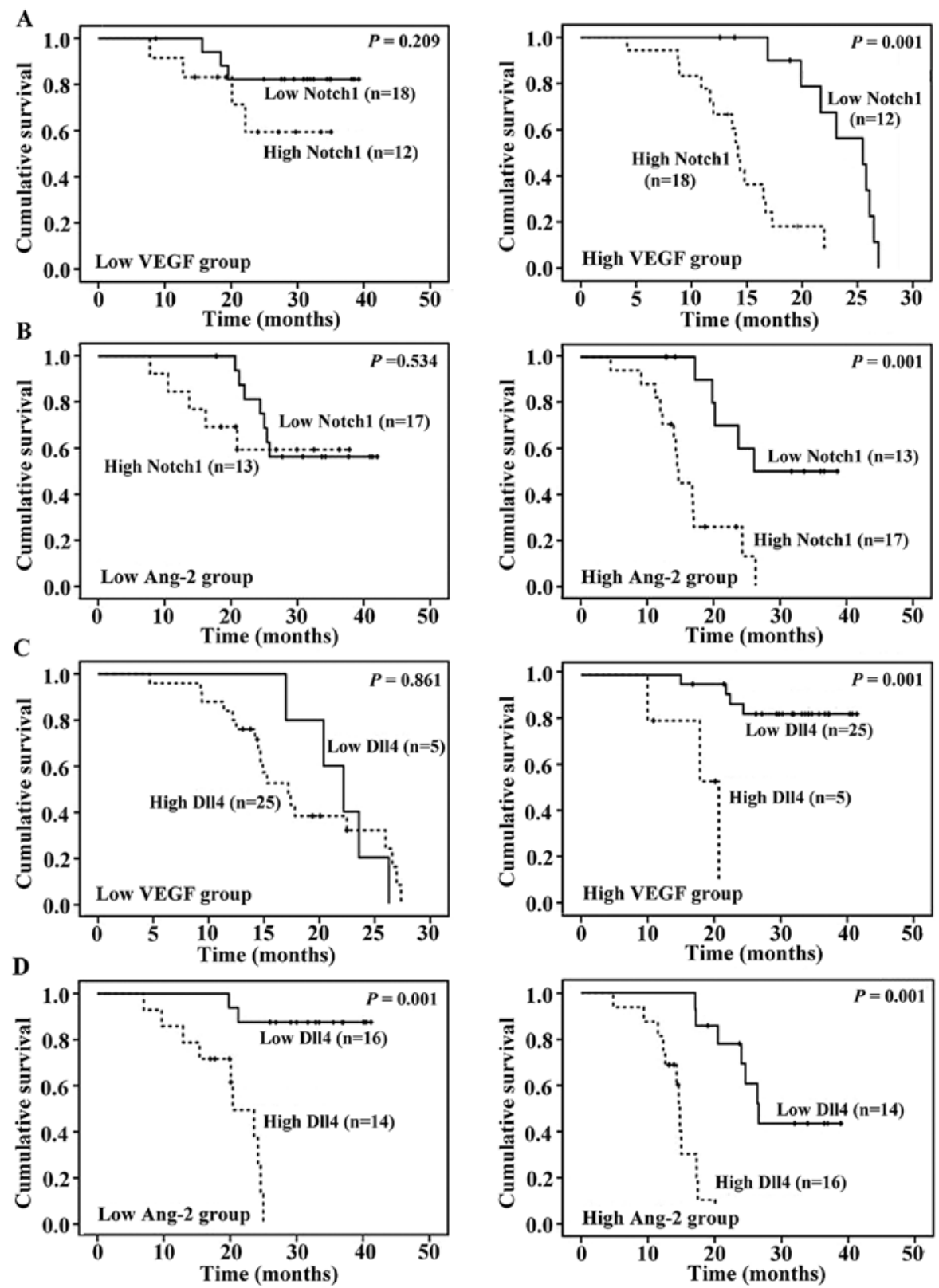

Figure 3. Subgroup analysis: Kaplan-Meier curves of overall survival stratified by Notch1 expression in the cohorts with low or high expression of (A) VEGF and (B) Ang-2, and by Dl14 expression in the cohorts with low or high expression of (C) VEGF and (D) Ang-2. Data were analyzed using the log-rank test.

that the alteration of the angiogenic balance in favor of Ang-2 acting in concert with VEGFR-2 may be essential for BM neovascularization in AML. However, we could not predict the prognosis of the AML patients based on the VEGFR2:VEGFR-1 and Ang-2:Ang-1 expression ratio.

In conclusion, $\mathrm{BM}$ angiogenesis in $\mathrm{AML}$ is a complex process involving the interplay of various angiogenic factors. Most investigators have attempted to elucidate the impact of a single angiogenic factor on the pathogenesis or prognosis of hematologic malignancies. Our present study not only investigated the prognostic role of the angiogenesis-related D1l4 and Notch1, but also assessed their association with the VEGF and Ang families. Our results provide evidence that the activation of the Notch/Dll4 pathway may indicate an unfavorable prognosis in AML. Furthermore, the role of the Notch/Dll4 pathway in AML angiogenesis and its correlation with VEGF and Ang-2 establishes the Notch/Dll4 pathway as a potentially important target of anti-angiogenic therapy. However, further prospective and multi-centre studies as well as in-depth studies are required to verify the role of Notch/Dll4 pathway in AML angiogenesis.

\section{Acknowledgements}

This study was supported by grants from the National Natural Science Foundation of China [81070422, 30871088, 81070407, 81000223]; the Specialized Research Fund for the Doctoral Program of Higher Education [20100131110060]; the Shandong Technological Development Project [2009HD012, 2009GG20002020, BS2009SW014, ZR2010HQ030] and the Independent Innovation Fund of Shandong University [yzc10072, yzc10075]. 


\section{References}

1. Zhang Y, Tang H, Cai J, et al: Ovarian cancer-associated fibroblasts contribute to epithelial ovarian carcinoma metastasis by promoting angiogenesis, lymphangiogenesis and tumor cell invasion. Cancer Lett 303: 47-55, 2011.

2. Hao CY: Angiogenesis blockade as therapy for hepatocellular carcinoma: progress and challenges. J Gastroenterol Hepatol 26: 4-6, 2011.

3. Shih TT, Hou HA, Liu CY, et al: Bone marrow angiogenesis magnetic resonance imaging in patients with acute myeloid leukemia: peak enhancement ratio is an independent predictor for overall survival. Blood 113: 3161-3167, 2009.

4. Trujillo A, McGee C and Cogle CR: Angiogenesis in acute myeloid leukemia and opportunities for novel therapies. J Oncol 2012: 128608, 2012

5. Payne SJ and Jones L: Influence of the tumor microenvironment on angiogenesis. Future Oncol 7: 395-408, 2011.

6. Ayala F, Dewar R, Kieran M and Kalluri R: Contribution of bone microenvironment to leukemogenesis and leukemia progression. Leukemia 23: 2233-2241, 2009.

7. Hou HA, Chou WC, Lin LI, et al: Expression of angiopoietins and vascular endothelial growth factors and their clinical significance in acute myeloid leukemia. Leuk Res 32: 904-912, 2008.

8. Carmeliet P and Jain RK: Molecular mechanisms and clinical applications of angiogenesis. Nature 473: 298-307, 2011

9. Wegiel B, Ekberg J, Talasila KM, Jalili S and Persson JL: The role of VEGF and a functional link between VEGF and $\mathrm{p} 27^{\mathrm{Kipl}}$ in acute myeloid leukemia. Leukemia 23: 251-261, 2009.

10. Schmidt T and Carmeliet P: Angiogenesis: a target in solid tumors, also in leukemia? Hematology Am Soc Hematol Educ Program 2011: 1-8, 2011

11. Tait CR and Jones PF: Angiopoietins in tumours: the angiogenic switch. J Pathol 204: 1-10, 2004.

12. Reikvam H, Hatfield KJ, Lassalle P, Kittang AO, Ersvaer E and Bruserud O: Targeting the angiopoietin (Ang)/Tie-2 pathway in the crosstalk between acute myeloid leukaemia and endothelial cells: studies of Tie-2 blocking antibodies, exogenous Ang-2 and inhibition of constitutive agonistic Ang-1 release. Expert Opin Investig Drugs 19: 169-183, 2010.

13. Maffei R, Martinelli S, Castelli I, et al: Increased angiogenesis induced by chronic lymphocytic leukemia B cells is mediated by leukemia-derived Ang2 and VEGF. Leuk Res 34: 312-321, 2010.

14. Keith T, Araki Y, Ohyagi M, et al: Regulation of angiogenesis in the bone marrow of myelodysplastic syndromes transforming to overt leukaemia. Br J Haematol 137: 206-215, 2007.

15. Aguayo A, Kantarjian HM, Estey EH, et al: Plasma vascular endothelial growth factor levels have prognostic significance in patients with acute myeloid leukemia but not in patients with myelodysplastic syndromes. Cancer 95: 1923-1930, 2002.

16. de Bont ES, Fidler V, Meeuwsen T, Scherpen F, Hahlen K and Kamps WA: Vascular endothelial growth factor secretion is an independent prognostic factor for relapse-free survival in pediatric acute myeloid leukemia patients. Clin Cancer Res 8 : 2856-2861, 2002.

17. Loges S, Heil G, Bruweleit M, et al: Analysis of concerted expression of angiogenic growth factors in acute myeloid leukemia: expression of angiopoietin-2 represents an independent prognostic factor for overall survival. J Clin Oncol 23: 1109-1117, 2005.

18. Schliemann C, Bieker R, Thoennissen N, et al: Circulating angiopoietin-2 is a strong prognostic factor in acute myeloid leukemia. Leukemia 21: 1901-1906, 2007.

19. Wu Y, Cain-Hom C, Choy L, et al: Therapeutic antibody targeting of individual Notch receptors. Nature 464: 1052-1057, 2010.

20. Demarest RM, Dahmane N and Capobianco AJ: Notch is oncogenic dominant in T-cell acute lymphoblastic leukemia. Blood 117: 2901-2909, 2011.

21. Leong KG and Karsan A: Recent insights into the role of Notch signaling in tumorigenesis. Blood 107: 2223-2233, 2006.

22. Phng LK and Gerhardt H: Angiogenesis: a team effort coordinated by notch. Dev Cell 16: 196-208, 2009.

23. Li JL and Harris AL: Notch signaling from tumor cells: a new mechanism of angiogenesis. Cancer Cell 8: 1-3, 2005.

24. Ridgway J, Zhang G, Wu Y, et al: Inhibition of Dll4 signalling inhibits tumour growth by deregulating angiogenesis. Nature 444: 1083-1087, 2006
25. Noguera-Troise I, Daly C, Papadopoulos NJ, et al: Blockade of Dll4 inhibits tumour growth by promoting non-productive angiogenesis. Nature 444: 1032-1037, 2006.

26. Lobry $\mathrm{C}, \mathrm{Oh} \mathrm{P}$ and Aifantis I: Oncogenic and tumor suppressor functions of Notch in cancer: it's NOTCH what you think. J Exp Med 208: 1931-1935, 2011.

27. Lobov IB, Brooks PC and Lang RA: Angiopoietin-2 displays VEGF-dependent modulation of capillary structure and endothelial cell survival in vivo. Proc Natl Acad Sci USA 99: 11205-11210, 2002.

28. Li JL and Harris AL: Crosstalk of VEGF and Notch pathways in tumour angiogenesis: therapeutic implications. Front Biosci 14: 3094-3110, 2009.

29. Thurston G and Kitajewski J: VEGF and Delta-Notch: interacting signalling pathways in tumour angiogenesis. Br J Cancer 99: 1204-1209, 2008.

30. Morrow D, Cullen JP, Cahill PA and Redmond EM: Ethanol stimulates endothelial cell angiogenic activity via a Notch- and angiopoietin-1-dependent pathway. Cardiovasc Res 79: 313-321, 2008.

31. Zhang J, Fukuhara S, Sako K, et al: Angiopoietin-1/Tie2 signal augments basal Notch signal controlling vascular quiescence by inducing delta-like 4 expression through AKT-mediated activation of beta-catenin. J Biol Chem 286: 8055-8066, 2011.

32. Kittang AO, Hatfield K, Sand K, Reikvam H and Bruserud O: The chemokine network in acute myelogenous leukemia: molecular mechanisms involved in leukemogenesis and therapeutic implications. Curr Top Microbiol Immunol 341: 149-172, 2010.

33. Muller-Berndorff H, Haas PS, Kunzmann R, Schulte-Monting J and Lubbert $\mathrm{M}$ : Comparison of five prognostic scoring systems, the French-American-British (FAB) and World Health Organization (WHO) classifications in patients with myelodysplastic syndromes: Results of a single-center analysis. Ann Hematol 85: 502-513, 2006.

34. Ji M, Li J, Yu H, et al: Simultaneous targeting of MCL1 and $\mathrm{ABCB} 1$ as a novel strategy to overcome drug resistance in human leukaemia. Br J Haematol 145: 648-656, 2009.

35. Lee CY, Tien HF, Hu CY, Chou WC and Lin LI: Marrow angiogenesis-associated factors as prognostic biomarkers in patients with acute myelogenous leukaemia. Br J Cancer 97: 877-882, 2007.

36. Ghaleb AM, Aggarwal G, Bialkowska AB, Nandan MO and Yang VW: Notch inhibits expression of the Kruppel-like factor 4 tumor suppressor in the intestinal epithelium. Mol Cancer Res 6: 1920-1927, 2008.

37. Li GH, Fan YZ, Liu XW, et al: Notch signaling maintains proliferation and survival of the HL60 human promyelocytic leukemia cell line and promotes the phosphorylation of the $\mathrm{Rb}$ protein. Mol Cell Biochem 340: 7-14, 2010.

38. Tohda S: Functional analysis of notch in the pathophysiology of leukemia. Rinsho Byori 57: 351-356, 2009 (In Japanese).

39. Donnem T, Andersen S, Al-Shibli K, Al-Saad S, Busund LT and Bremnes RM: Prognostic impact of Notch ligands and receptors in nonsmall cell lung cancer: coexpression of Notch-1 and vascular endothelial growth factor-A predicts poor survival. Cancer 116: 5676-5685, 2010.

40. Reedijk M, Odorcic S, Chang L, et al: High-level coexpression of JAG1 and NOTCH1 is observed in human breast cancer and is associated with poor overall survival. Cancer Res 65: 8530-8537, 2005.

41. Shi TP, Xu H, Wei JF, et al: Association of low expression of notch-1 and jagged-1 in human papillary bladder cancer and shorter survival. J Urol 180: 361-366, 2008.

42. Siekmann AF, Covassin L and Lawson ND: Modulation of VEGF signalling output by the Notch pathway. Bioessays 30: 303-313, 2008.

43. Williams CK, Li JL, Murga M, Harris AL and Tosato G: Up-regulation of the Notch ligand Delta-like 4 inhibits VEGFinduced endothelial cell function. Blood 107: 931-939, 2006.

44. Wakabayashi M, Miwa H, Shikami M, et al: Autocrine pathway of angiopoietins-Tie2 system in AML cells: association with phosphatidyl-inositol 3 kinase. Hematol J 5: 353-360, 2004.

45. Xu Q, Simpson SE, Scialla TJ, Bagg A and Carroll M: Survival of acute myeloid leukemia cells requires PI3 kinase activation. Blood 102: 972-980, 2003. 